\title{
Personalization of user Profile: Creating user Profile Ontology for Tamilnadu Tourism
}

\author{
K.R.Ananthapadmanaban, \\ Research Scholar, \\ Sri Chandrasekarendra SaraswathiViswa \\ Mahavidyalaya University, \\ Enathur, Kanchipuram-631561
}

\author{
Dr.S.K.Srivatsa \\ Senior Professor, \\ St. Joseph's College of Engg, \\ Jeppiaar Nagar, \\ Chennai-600 064
}

\begin{abstract}
The semantic web enabled information retrieval aims at making the information flooding in the World Wide Web accessible to more precise search and information extraction based machine readable representation. The applications that deliver these new online solutions are based on ontology. This paper explores creating sophisticated user profiles ontology which can improve the process of searching for the perfect tourism package by analyzing the user interest with help of user ontology for Tamilnadu tourism.
\end{abstract}

\section{General Terms}

Semantic Web

\section{Keywords}

Semantic Web, Ontology, Protégé Tool, OWL

\section{INTRODUCTION}

When surfing on the Internet, end users are increasingly in need of more powerful tools capable of searching and interpreting the vast amount of heterogeneous information available on the web. Current Web has been designed for direct human processing, but the next generation "semantic Web," aims at machine process able information [4]. Retrieval of keyword and text matching in the retrieval engine can be successful only through Semantic Web [2] [11]. Ontologies and ontology-based information retrieval have the potential to significantly improve the process of searching information on the World Wide Web.

Ontology is a hierarchy of the most important concepts related to a particular domain, their relationships and their properties [10] [15]. Ontologies describe vocabularies as a kind of complex (meta-) data schemata that are used in order to combine semantic meta data and offer added-value services on the top of semantic descriptions. This paper focuses on creating sophisticated user profile ontology which can improve the process of searching for the perfect Tourism package by analyzing the user interest with the help of user ontology for Tamilnadu tourism.

\section{LITERATURE SURVEY}

Ontology is an explicit, formal specification of a shared conceptualization [8]. "Conceptualization" means an abstract, simplified view of the world. The world actually refers to some phenomenon, topic, or subject area in the world. Every representation of the knowledge in an area of interest is based on a conceptualization. Every conceptualization is grounded on the concepts, objects, and the relationships among them [9].

\begin{abstract}
"Explicit" means that the type of concepts and the constraints on using the concepts are explicitly defined in the data structure of ontology. "Formal" indicates that the ontology should be machine interpretable. "Specification" means a declarative representation of the knowledge. "Shared" implies that ontology is not supposed to represent the subjective knowledge of some individual, but it captures consensual knowledge accepted by a group or a community. In short, ontology is the manifestation of a shared understanding of a domain that acquired a consensus, and such consensus can facilitate effective communications. This, in turn, leads to other benefits such as interoperability, reuse and sharing [1] [7].
\end{abstract}

Ontologies can be developed in different languages like RDF, RDF SCHEMA, XML, XML Schema and OWL. The later is most widely used compared to other languages. OWL is a set of XML elements and attributes with well defined meaning that are used to define terms and their relationship (e.g. class, equivalent property, intersection Of, union Of, etc..,). Owl representation is a language with sufficient expressive and reasoning power to represent the semantic of ontology.

Tim Berners-Lee pointed out that Semantic Web has all the standards and technologies it needs to succeed and that it was time for Web developers and content producers to start using semantic languages in addition to HTML [3]. Using Semantic Web technologies in the area of tourism can improve already existing systems (which are mostly available online) that do not use Semantic Web techniques yet. Likewise, the Semantic Web approach can help decrease the maintenance efforts required for existing E-Tourism systems and ease the process of searching for vacation packages. Jakkilinki. R., Georgievski.M. and Sharda.N. present a tool for tour planning that is intelligent in the meaning that it generates travel plans by matching user preferences and available tourist offers from different travel agents in respect to the ontology which enables reasoning [12]. Eleni Tomai, Stavros Michael, and Poulicos Prastacos explored how to use ontologies and constructed two ontologies one for user profile and another one concerning tourism information and data in order to assist visitors of an area to plan their visit [6]. Danica Damljanovic, University of Sheffield, UK and Vladan Devedzic, University of Belgrade, Serbia illustrated that developing more sophisticated user profiles would help developing more personalised system thus avoiding spaming the user with an unattractive content [5].

On observations made in the literature review, Tourism is not a group with homogeneous characteristics, the notation of personalisation is crucial in the design, User profiles are 
application specific (i.e. each one having been created specifically for a particular domain) and the user profiles should be location based. In this paper we are going to construct user profile ontology for Indian scenario especially with parameters for Tamilnadu tourism to assist visitors to plan their trip accurately.

\section{ONTOLOGY DEVELOPMENT}

\subsection{Design steps}

1. The admin or ontology engineer creates ontology by using Protégé tool.

2. If any activity is to be added to the ontology the ontology needs to be updated. The ontology engineer updates the ontology by adding instances.

3. End user searches for web content and issues requests using GUI.

4. The End user query to Jena agent and ontology will be traversed according to end user specification.

5. The Jena agent retrieves the query result and passes the result to GUI.

The ontology has been developed using protégé 4.1 beta version for travel and tourism domain. The key focus of this paper is creation of sophisticated user-profile ontology to enable personalization of web content in tourism domain. The screenshots of user profile form (interface) is also included for better clarity.

\subsection{Implementation details}

Protégé is an open source tool which is used to construct knowledge based application using ontology. It provides a platform for creating ontology and form the ontology knowledgebase. The tool displays and edits ontology in graphical mode. It also helps in building OWL-DL ontology and using a Description Logic Reasoner to check the consistency of the ontology and automatically compute the ontology class hierarchy.

\subsection{User profile ontology creation}

The user profile ontology was created in order to facilitate the extraction of the user personal information, needs and interests under the context of personalisation. The user is requested to register and fill information in few forms with personal information such as accompanying persons in travel i.e. if he is travelling alone, or with family or friends or as couple. No. of persons accompanying and their age group is classified by asking no. of children, adults, teenagers and persons aged above 60 travelling with him/her. Their area of interest in tourism such as historical places, Beaches, Hill Stations, Zoological Parks, Botanical Gardens etc., is also collected, user leisure activities like Swimming, Cycling, Golf etc., is also identified through an interface. The user is also requested to provide trip date. Based on the information about accompanying persons with the user, their area of interest perfect holiday destination to the user can be identified. For identifying perfect holiday destination requires intelligent reasoning and it is necessary to build and maintain appropriate knowledge in the system.

To be more specific user's interests is classified as Historical Heritage, Sightseeing, Nature and Spiritual. The Historical and

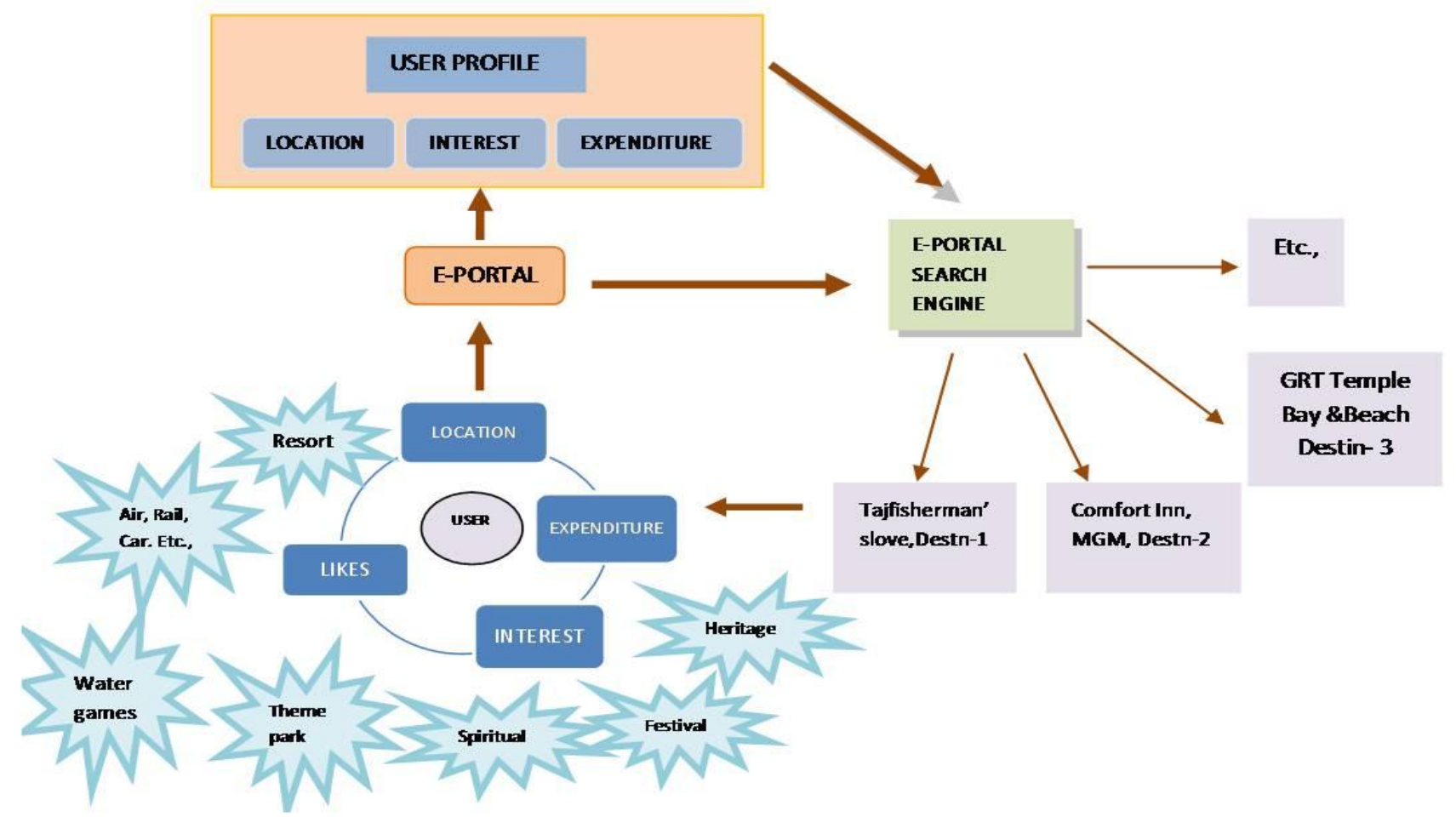

Figure 1-Adaptive Portal using User Profile 
Heritage is further classified as Monument, Memorial, Art Gallery, Palace and Fort. Similarly Sightseeing is classified as Beach, Hill Station, Waterfall and Festival. Nature is classified as Wildlife Zoological Park, Sanctuary and Botanical Garden. And Spiritual is further classified as Temple and Church. These were further supported by adding concepts such as Likes, Activity, Transportation and EatingOut. For instance, for the concept Likes, the sub-concepts Antique Shopping, Jewellery Shopping, Mall, Super Market, Theme Park, Bars and Discotheque and Fine Arts were created. The Figure 2 and 3 show the class description using protégé and plug-in in OWL Viz. Figure 4 shows full class descriptions of user ontology using Onto Graf.

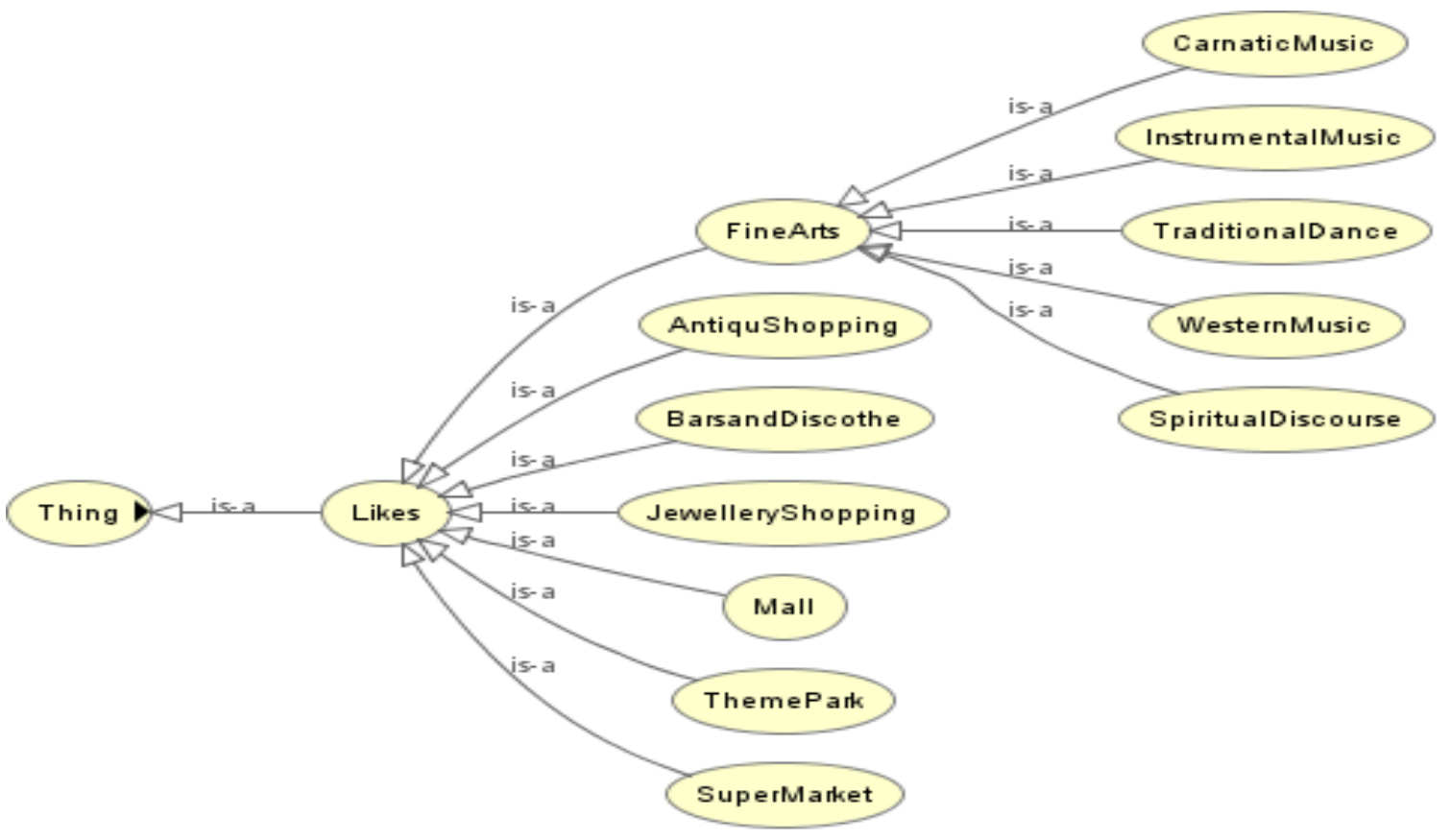

Figure 2-Class Description of Likes using OWL Viz.

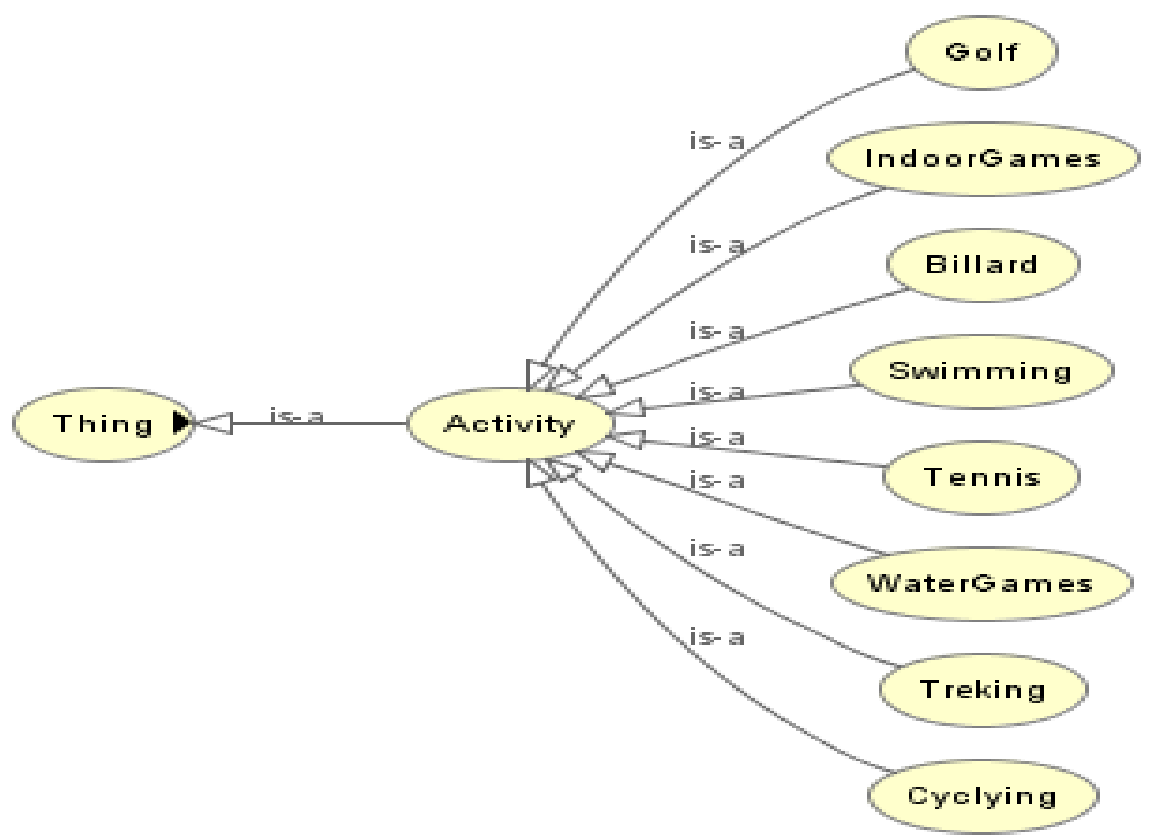

Figure 3-Class Description of Activity using OWL Viz. 


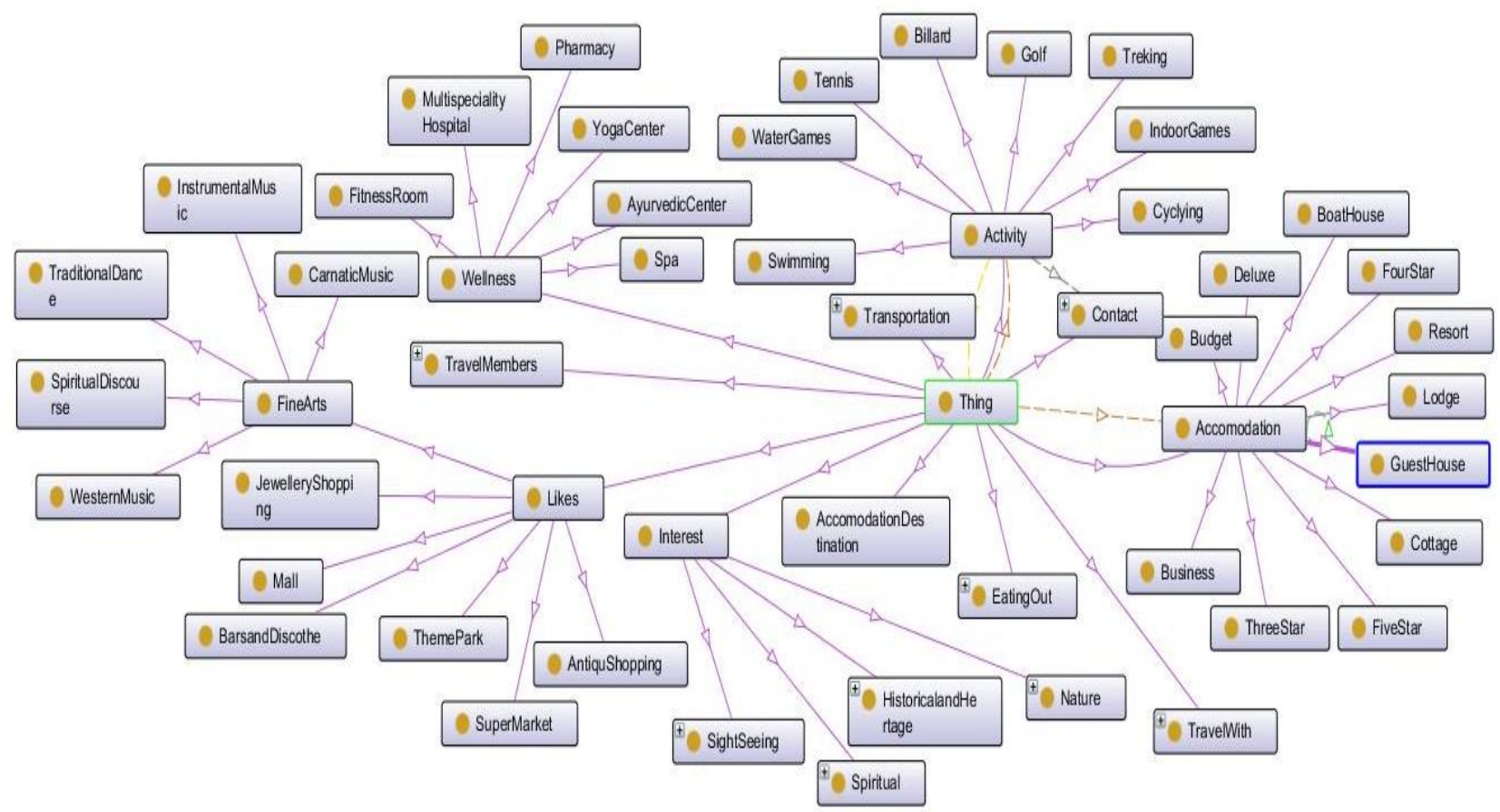

Figure 4-Class description of user Ontology using OntoGraf.

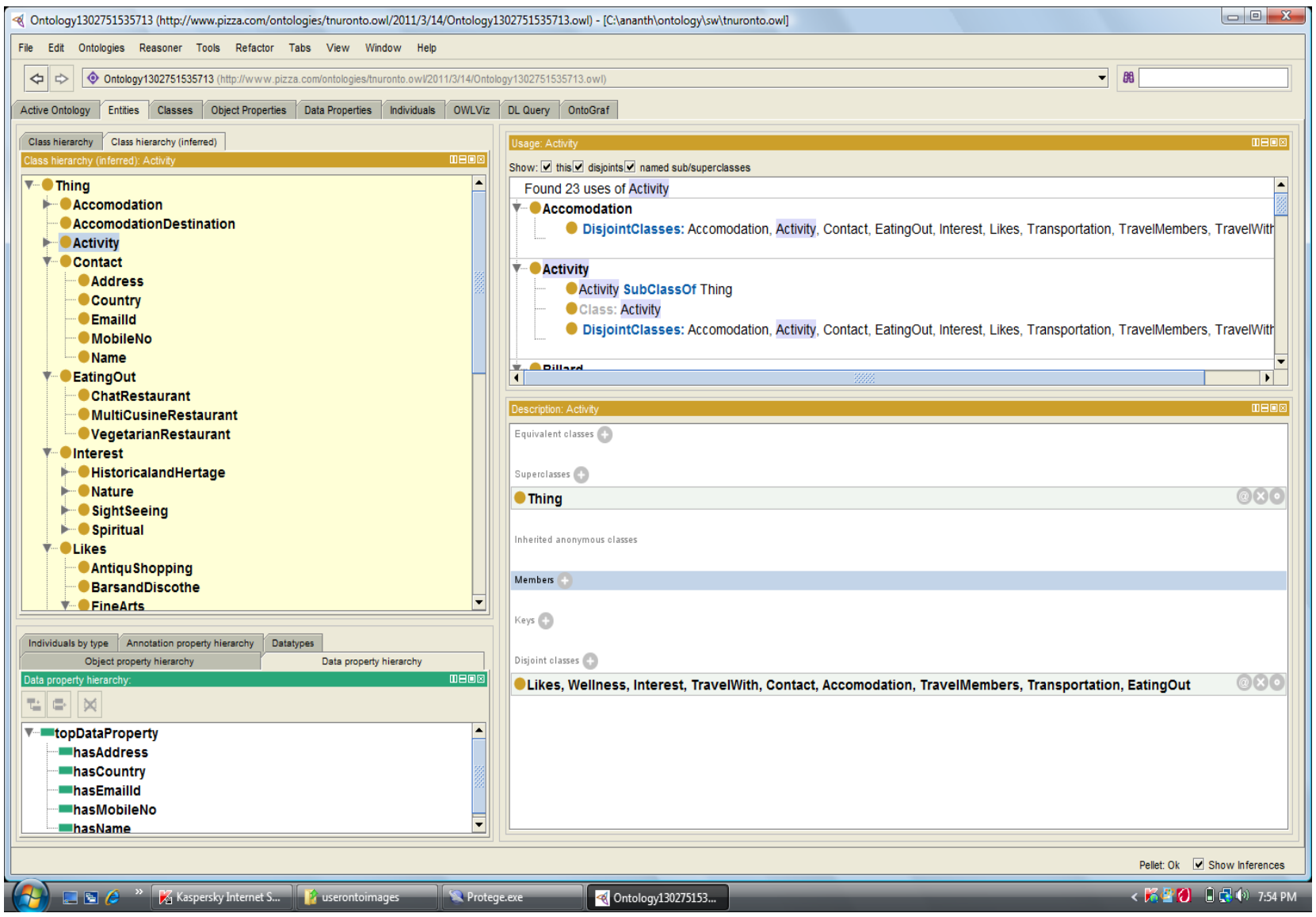

Figure 5- A Screen shot of the Protégé Ontology development environment 
In Fine Arts, the sub-concepts Instrumental Music, Carnatic Music, Traditional Dance, Western Music and Spiritual Discourse were created. Based on the each concept a corresponding property was created. There are two types of properties namely Object property and Data type property. The Data type property mentions the data type for each property. While creating ontology property with corresponding subclass, range and allowable values for that property to be specified. The Object property mentions the relationship between each class and concept. We can create number of instances or individuals for each class or concept and assign values for each instance based on data type property. By the help of interface (User profile form) user profile ontology can be populated and information like user's personal data, interests, likes and eating habits can be collected and recorded. The user's appropriate destination can also be identified with the help of user profile ontology.

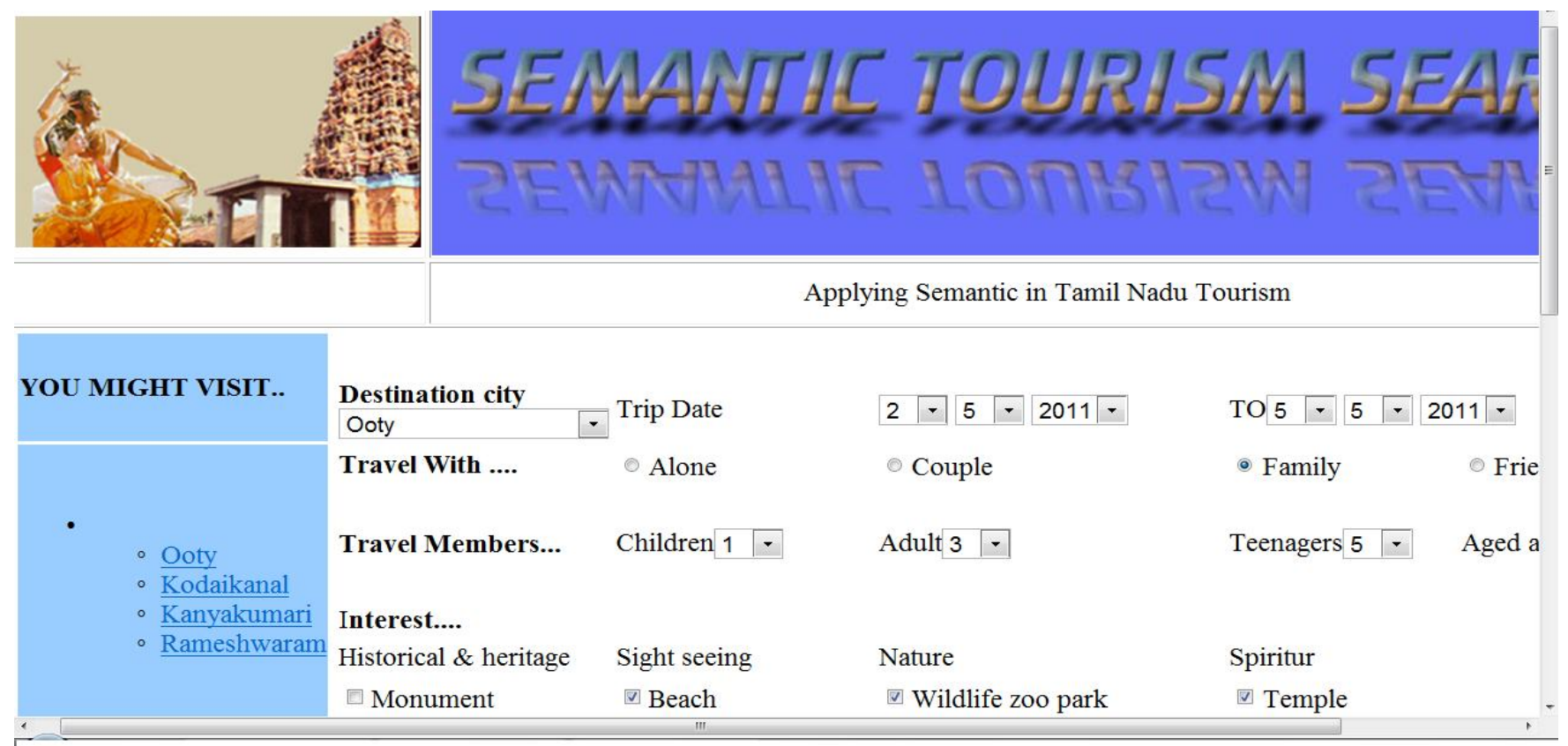

Figure 6-Screenshots of UserProfile-1

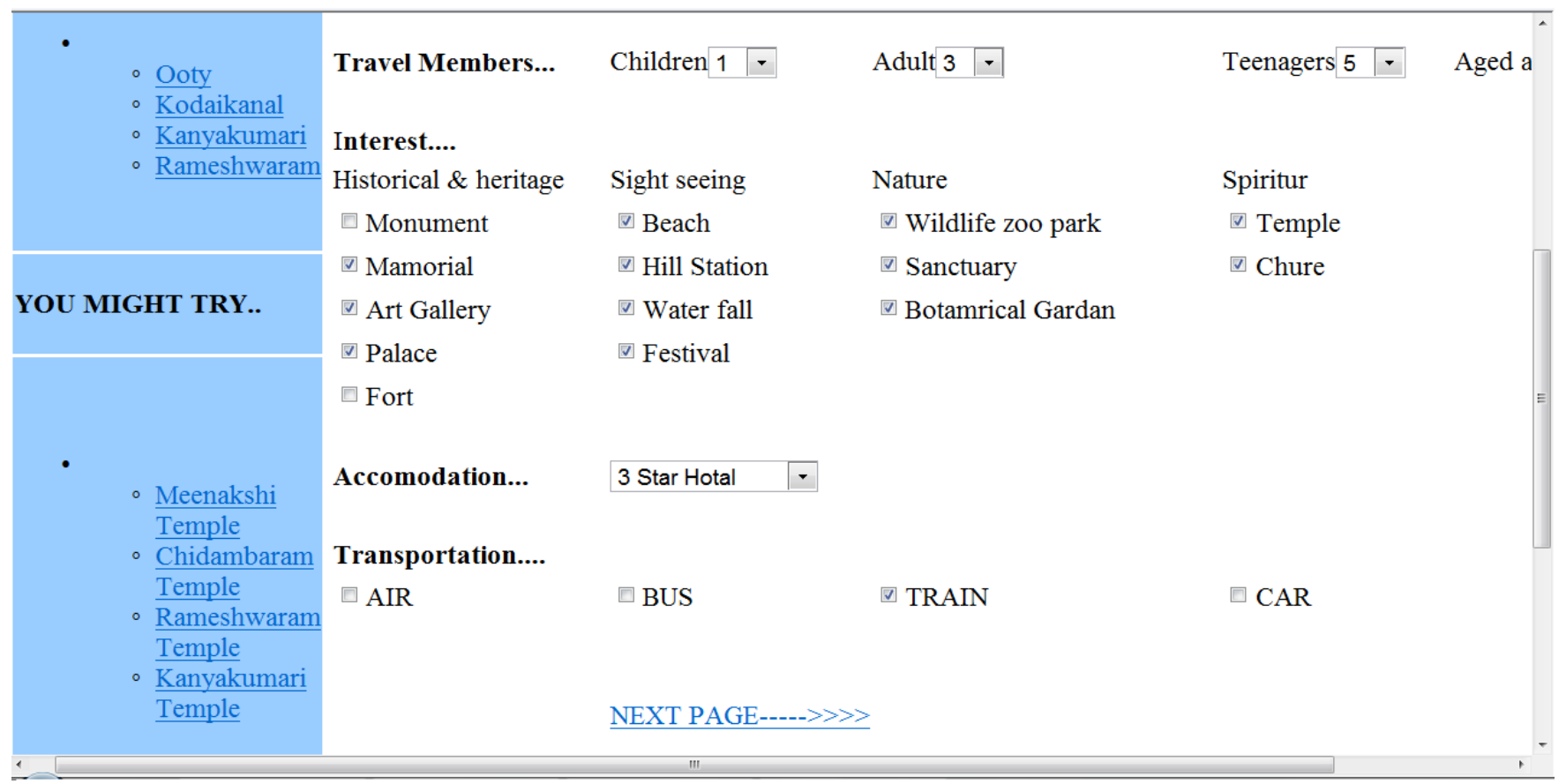

Figure 7-Screenshots of UserProfile-2 


\section{CONCLUSION}

It could be observed that the tourism sector is an area in which ontologies can be applied anywhere. In this paper we have created user profile ontology through which we can infer tourist specific area of interest. Ontology based search can be made on tourism sites by constructing tourism ontology and information can be generated accurately according to the tourist interest using user profile ontology.

\section{FUTURE WORK}

Integrating semantic web technologies in existing web applications as a vast space for improvement. Employing ontologies is the most advisable way as they enable data in a machine readable form. In this paper, we have created the user ontology for tourism in Tamilnadu.

Similarly Tourism ontology to be created and by means of an interface user can fill the user profile and the user profile ontology can be automatically populated. Based on the user profile and tourism ontology inferences can be made and more accurate results with higher level of accuracy can be obtained and appropriate tourist spots of user interest in Tamilnadu can be generated.

\section{REFERENCES}

[1] Agarwal, P., 2005, Ontological considerations in GIScience, International Journal of Geographical Information Science, 19, 501-536.

[2] Alexander Maedchea and Steffen staabb, "Applying Semantic Web Technologies for Tourism Information Systems", Proceedings of International conference on IICT in Tourism, 2002, Innsbruck, Austria

[3] [Online].Retrieved January 3, 2007from:http://networks.silicon.com/webwatch/0,390248 76,39159122,00.htmTavel, P. 2007 Modeling and Simulation Design. AK Peters Ltd.

[4] T.Berners-Lee, J.Hendler, and O.Lasilla, 2001, "The Semantic Web", Scientific American.
[5] Danica Damljanovic, University of Sheffield, UK and Vladan Devedzic, University of Belgrade, Serbia. "Applying Semantic Web to E-Tourism" 2009.

[6] Eleni Tomai, Stavros Michael, and Poulicos Prastacos, Forth Institute of Applied Computational Mathematics Heraklion Greece-“An Ontology-based Web-portal for Tourism".

[7] Gaševi'c, D., Djuri'c, D., and Devedz'i'c, V., 2006, "Model Driven Architecture and Ontology Development", Springer, Berlin-Heidelberg.

[8] Gruber, T. R., 1993, A translation approach to portable ontologies, Knowledge Acquisition, 5, 99-220.

[9] Genesereth, M. R. and Nilsson, N. J., 1987, Logical Foundations of Artificial Intelligence.

[10] Guarino,N.,Carrara,M.,Giaretta,P.; “An ontology of metaleveled categories"

[11] Hannes Werther, "Intelligent Systems in Travel and Tourism", IEEE Intelligent Systems, Volume 17, Issue 6 November 2003.

[12] Jakkilinki, R., Georgievski, M. \& Sharda, N. (2007). "Connecting Destinations with an Ontology- Based eTourism Planner". In Sigala, M., Mich, L. \& Murphy, J. (Eds.): Information and Communication Technologies in Tourism 2007, Proceedings of the International Conference in Ljubljana, Slovenia, 2007. (pp. 21-31). Springer Vienna.

[13] Maria Golemati, Akrivi Katifori, Costas Vassilakis, George Lepouras, Constantin Halatis, "Creating Ontology for User profile: Method and Applications".

[14] The Protégé project http://protege.stanford.edu,002 on Knowledge and Data Engineering, Vol. 19, No. 2, February 2007.

[15] Staab, S., Studer, R.Editor; "Handbook on Ontologies", Springer, 2004. 